\title{
A saúde como objeto de consumo: uma análise sobre as demandas e consultas públicas de incorporação de medicamentos no SUS.
}

Health as an object of consumption: an analysis on the demands and public consultations of incorporation of medicines in the Brazilian Health System - SUS

La salud como un objeto de consumo: un análisis de las demandas y consultas públicas sobre medicamentos en el SUS

\section{Luanna de Mendonça Gomes ${ }^{1}$}

Resumo: Este artigo pretende analisar as demandas por incorporação de medicamentos no Sistema Único de Saúde (SUS), encaminhadas à avaliação da Comissão Nacional de Incorporação de Tecnologias no SUS (CONITEC). O objetivo geral desse estudo é identificar os principais segmentos sociais que participam do processo de incorporação de medicamentos no SUS e refletir sobre as possíveis influências desses segmentos no processo de incorporação de novas tecnologias em saúde. O estudo foi realizado por meio de uma pesquisa descritiva quantitativa das informações disponíveis no site da CONITEC sobre as demandas e as consultas públicas realizadas nos anos de 2012, 2013 e 2014 sobre incorporações de medicamentos no SUS. Os resultados obtidos revelam que empresas e laboratórios farmacêuticos são responsáveis por 44\% das demandas realizadas no período. O Ministério da Saúde é o segmento que demanda revisões para exclusão de tecnologias, e possui o maior número de solicitações que resultam em incorporação $(66 \%$ do total de incorporações entre 2012 e 2014). Os demais segmentos possuem baixa representatividade nas demandas e dificuldades no cumprimento das conformidades documentais exigidas pela CONITEC. Nas consultas públicas, as empresas e laboratórios farmacêuticos são o segmento mais participativo e foi constatada uma baixa participação de usuários do SUS e profissionais de saúde. Conclui-se que o processo de incorporação de medicamentos no SUS necessita de maior participação social, principalmente dos usuários e que conhecimento técnico especializado nas avaliações de tecnologias não devem se constituir numa justificativa para a não inserção da sociedade nesse processo.

Palavras-chaves: Incorporação de Medicamentos, Assistência Farmacêutica, Tecnologias em Saúde

Abstract: This article aims to analyze the demands on incorporation of drugs in SUS sent to the evaluation of the National Committee for the Incorporation of Technology in the SUS (CONITEC). The overall objective of this study is to identify the major social groups that participate in the embedding process of drugs in the SUS and the reflection on the possible influence from these segments in the incorporation of new technologies in health care process. The study was conducted through a descriptive and quantitative research using the available information in CONITEC about the demands and public consultations carried out in the years 2012, 2013 and 2014 on incorporation of drugs in SUS. The results reveal that businesses and pharmaceutical companies are responsible for $44 \%$ of demands for the period. The Ministry of Health is the segment that requires revisions to the exclusion of

${ }^{1}$ Especialista em Direito Sanitário. Especialista em Gestão de Saúde. Farmacêutica do Laboratório Central de Saúde Pública da Secretaria de Estado de Saúde do Distrito Federal. Brasília - DF, Brasil. 
technologies and has the largest number of requests that resulted in incorporation $(66 \%$ of all mergers between 2012 and 2014). Other segments have a low representation in the request, and have difficulties in complying with the formal compliances required by CONITEC. In Public consultations, the pharmaceutical companies are the most participatory segment. The SUS users and health professionals have a poor participation on this research. It is concluded that the process of incorporation of drug in SUS requires greater social participation, especially of the users of the system. Furthermore, the technical expertise in the analysis process of technologies should not constitute a justification for not inserting the society in the process.

Keywords: Incorporation of Drugs, Pharmaceutical Assistance, Technologies in Health.

Resumen: Este artículo analiza las demandas por incorporación de los medicamentos en el Sistema Único de Salud (SUS), remitida a la evaluación de la Comisión Nacional de Tecnología Incorporación en el SUS (CONITEC). El objetivo general de este estudio es identificar los principales grupos sociales que participan en el proceso de incorporación de los fármaco en el SUS y sobre las posibles influencias de los segmentos farmacéuticos en el proceso de incorporación de las nuevas tecnologías en salud. El estudio se llevó por medio de una investigación descriptiva cuantitativa de la información disponible en el sitio de la CONITEC sobre las demandas y consultas públicas en los años 2012, 2013 y 2014 sobre la incorporación de medicamentos en el SUS. Los resultados muestran que las compañías farmacéuticas representan $44 \%$ de la demanda en el período. El Ministerio de Salud es el segmento que requiere revisiones para tecnologías de eliminación, y tiene el mayor número de solicitudes que se traducen en la incorporación (66\% del total de las fusiones entre 2012 y 2014). Los otros segmentos tienen una baja representación en las exigencias y dificultades en el cumplimiento de los incumplimientos documentales exigidos por CONITEC. En las consultas públicas, y las compañías farmacéuticas son se encontró segmento más participativa y una baja participación de los usuarios del SUS y profesionales de la salud. Llegamos a la conclusión de que el proceso de incorporación del fármaco en el SUS necesita más participación social, especialmente de los usuarios y los conocimientos técnicos en las evaluaciones de tecnología no debe constituir una justificación para la no inclusión de la sociedad en este proceso.

Palavras-llaves: Incorporación de las Drogas, Asistencia Farmacéutica, Tecnologías en Salud

\section{Introdução}

O direito à saúde no Brasil foi positivado como um direito fundamental e dever do Estado, garantido mediante a implementação de políticas públicas que permitam o acesso universal e integral às ações e serviços do Sistema Único de Saúde por meio da Constituição Federal de 1988 (1).

O conceito de acesso integral à saúde é compreendido, muitas vezes, exclusivamente como o acesso às tecnologias em saúde. As tecnologias em saúde devem ser o meio que permite a prestação do cuidado e da atenção à saúde da população (2). Essas tecnologias 
incluem equipamentos médicos, produtos para saúde, medicamento, vacinas, testes diagnósticos, órteses e próteses, materiais e sistemas informacionais de aplicação na assistência à saúde (3).

\section{O complexo produtivo e industrial da saúde e as demandas por novas tecnologias para os sistemas de saúde}

O complexo produtivo e industrial da saúde é uma das áreas de grande crescimento econômico no mundo. A inovação em saúde tem sido demandada por uma série de fatores, tais como o envelhecimento da população, a necessidade de tratamentos especializados, dentre outros (3).

Para Capucho et al, (2), não há dúvidas de que os avanços das pesquisas e desenvolvimento de novas tecnologias em saúde são benéficos para a população. Porém, é cada vez maior a vulnerabilidade dos sistemas universais de saúde frente às dificuldades de incorporação de tecnologias essenciais para a promoção, a prevenção e a atenção à saúde. Para Gadelha (4):

O processo técnico, reconhecido como base do desenvolvimento desde Adam Smith, Marx e Schumpeter, e todos os pensadores estruturalistas, como Celso Furtado, traz, simultaneamente, o risco de cindir a sociedade e acirrar a desigualdade entre os que têm acesso e os que não têm acesso às novas tecnologias.

Além disso, todas as tecnologias trazem benefícios, riscos e custos para a sociedade; e para disponibilização de uma tecnologia em um sistema de saúde todos esses aspectos devem ser minimamente avaliados (2). Um importante gargalo desse processo é que os recursos são escassos, e que por restrições orçamentárias, os sistemas universais de saúde podem não conseguir oferecer todas as intervenções tecnológicas disponíveis no mercado. Para isso, as avaliações de tecnologias devem fornecer mecanismos para que haja comparação entre a nova tecnologia demandada à incorporação e as que já estão disponíveis no sistema de saúde, com base em estudos de custo-efetividade (5).

A pressão por novas tecnologias no Sistema Único de Saúde se manifesta de várias formas e por diversas demandas que envolvem a ação de produtores, pacientes, prescritores, sociedades médicas, associações de portadores de patologias e do judiciário. A modernização do sistema de saúde é objeto de desejo da sociedade e por muitas vezes 
sem preocupação alguma com a racionalização e a segurança que deve ser inerente ao processo de inovação tecnológica (3).

Segundo a Política Nacional de Gestão de Tecnologias em Saúde (6), os sistemas de saúde em todo o mundo nas últimas décadas, ainda que não inseridos em economias de mercado, foram bastante influenciados por políticas públicas com várias perspectivas inclusive de fortalecimento dos seus usuários e profissionais de saúde, que juntos exercem uma forte pressão por incorporação de novas tecnologias.

A política ainda aborda que o crescimento contínuo dos gastos em saúde, a produção cada vez maior de tecnologias e as alterações do perfil epidemiológicos da população levam a necessidades diversificadas de atenção à saúde. E dessa forma, faz-se necessário tanto social quanto politicamente o desenvolvimento de mecanismos de articulação entre os setores envolvidos na produção, incorporação e na utilização dessas tecnologias nos sistemas de saúde (6).

\section{A Avaliação de Tecnologias em Saúde como método para as análises das demandas por incorporação de medicamentos no SUS}

Para que se possa incorporar uma nova tecnologia em saúde, deve-se compreender esse processo como uma área de conhecimento interdisciplinar que além de considerar aspectos éticos, deve possuir embasamento técnico em revisão sistemática, crítica e criteriosa da literatura disponível (2). A área de avaliação de tecnologias na área da saúde, como área de conhecimento e práticas, teve início nos anos 70 no Estados Unidos, vinculadas às atividades legislativas e se desenvolveu na Europa como parte da gestão dos sistemas de saúde, principalmente nos países de sistemas universais e públicos, como na Suécia, Holanda e Reino Unido (7).

Atualmente, essa ferramenta ganhou espaço em comissões ou serviços de assessoria em instâncias de diversos setores do setor público, nas universidades, e também nas associações profissionais e empresas privadas. Capucho et al. (2) ressaltam que a incorporação de tecnologias tem importantes implicações políticas, econômicas, administrativas e sanitárias, especialmente em sistemas de saúde como o do Brasil.

Com a designação constitucional do papel do estado em prover o direito integral à saúde, a judicialização tem desafiado o princípio da equidade e de certa forma ameaçando o sistema de saúde, deslocando prioridades e gerando riscos. Todavia, possui o mérito de 
sinalizar o que a sociedade esperar do SUS. O grande desafio da avaliação das tecnologias em saúde a serem incorporadas está na capacidade de observar critérios de eficácia, acurácia, segurança, análise econômica e sua real contribuição para a promoção, manutenção ou reabilitação da saúde; bem como obedecer a critérios de priorização, oportunidade, adequação ou racionalidade, a fim de beneficiar a sociedade (2).

\section{A Saúde como um sonho de consumo e seu reflexo no Sistema Único de Saúde}

A saúde se tornou um objeto de consumo na sociedade economicamente emergente e as empresas do mundo inteiro descobriram isso (8). Para Chomsky (9), não se pode perder de vista que o desenvolvimento tecnológico se volta mais para os interesses mercadológicos, para o lucro, considerando que todo cidadão é consumidor de saúde. $\mathrm{O}$ Poder Público deve atuar frente às pressões de mercado instituindo políticas de gestão do conhecimento, visando fomento à pesquisa, à sistematização do conhecimento nos serviços de saúde, a avaliação e divulgação do conhecimento e à incorporação das inovações tecnológicas nos serviços de saúde (8).

Para Lenir Santos (8), as tecnologias em saúde têm sido aplicadas de modo individualizado, desconsiderando a política de saúde em sua forma sistêmica, o que pode inviabilizar o Sistema Único de Saúde, fracionando-o de tal maneira que o acesso às tecnologias só será concedido a quem demandar, em especial no âmbito do Judiciário e sem a devida racionalidade.

Ainda para Lenir Santos (8):

Sem uma política adequada de avaliação de tecnologia e fomento à pesquisa em saúde, estaremos à deriva, colocando em risco o sistema de saúde que se funda no padrão de integralidade da assistência, que por sua vez, bebe da fonte da incorporação de tecnologias.

O Supremo Tribunal Federal encaminhou uma recomendação ao Ministério da Saúde propondo melhores definições normativas sobre a questão da incorporação tecnológica, exigindo, pois, uma adequada gestão da incorporação tecnológica. E dessa forma, após uma audiência pública sobre saúde, em 2009, foi aprovada a Lei no 12.401 de 28 de abril de 2011, que altera a Lei 8.080/90, para dispor sobre a assistência terapêutica e a incorporação de tecnologia em saúde no âmbito do Sistema Único de Saúde (2). 


\section{A Comissão Nacional de Incorporação de Tecnologias no SUS (CONITEC) e o processo de incorporação de medicamentos no SUS}

A CONITEC foi criada pela Lei no 12.401 , de 28 de abril de 2011, como um espaço colegiado institucionalizado para assessorar o Ministério da Saúde na incorporação, na exclusão ou na alteração pelo SUS de novos medicamentos, produtos e procedimentos, bem como na constituição ou na alteração de protocolo clínico ou de diretriz terapêutica (10).

A regulamentação de seu funcionamento foi publicada por meio do Decreto 7.646 , de 21 de dezembro de 2011, que define a estrutura da CONITEC que contempla um Plenário e uma Secretaria Executiva. O Plenário é o fórum responsável pela emissão de recomendação sobre incorporação, exclusão ou alteração das tecnologias no âmbito do SUS, sobre constituição ou alteração de protocolos clínicos e diretrizes terapêuticas e atualização da Relação Nacional de Medicamentos Essenciais (RENAME), enquanto que a Secretaria Executiva é responsável pela gestão e coordenação das atividades da comissão, bem como a emissão de relatórios técnicos sobre a tecnologia avaliada, levando em consideração as evidências científicas, a avaliação econômica e o impacto da incorporação da tecnologia no SUS (11).

O Plenário é constituído por 13 membros, sendo estes representantes das secretarias do Ministério da Saúde, do Conselho Nacional de Saúde, do Conselho Nacional dos Secretários Estaduais de Saúde, do Conselho Nacional de Secretarias Municipais de Saúde e do Conselho Federal de Medicina (12). Para Capucho et al (2), a participação social está contemplada no processo de tomada de decisão e foi reforçada na legislação atual, pois prevê a representação do Conselho Nacional de Saúde na comissão e também por garantir a obrigatoriedade da realização das consultas públicas.

A legislação também determina que a disponibilização à população brasileira das tecnologias incorporadas deve ser realizada num prazo máximo de 180 dias (10). O fluxo básico do processo de incorporação está disposto na figura 1 . 
Figura 1 - Fluxo Simplificado de incorporação de tecnologias no SUS
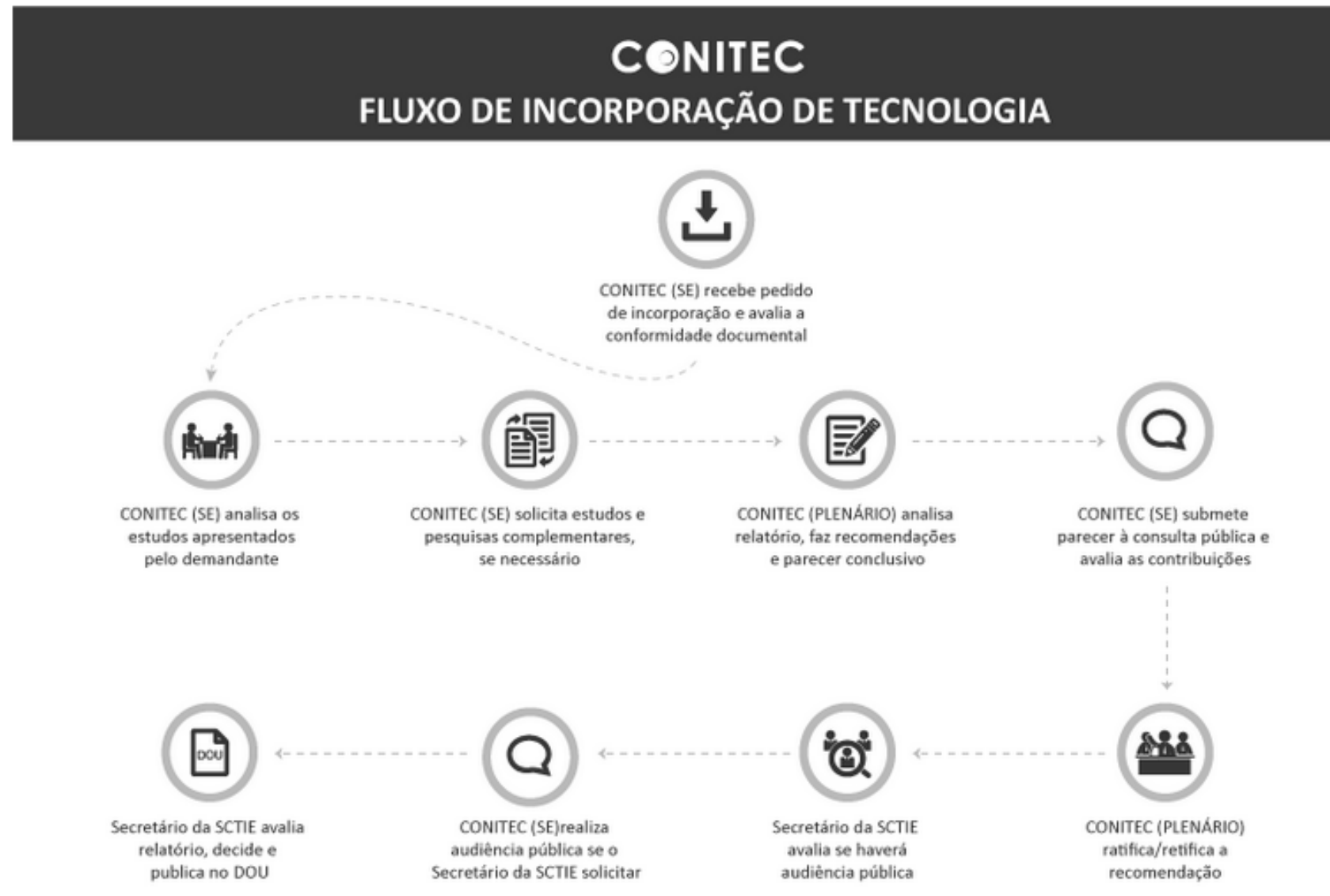

Fonte: CONITEC, 2014.

\section{A criação da CONITEC e o direito sanitário}

A instituição da Comissão Nacional para Incorporação de Tecnologias no SUS (CONITEC), em 2011, se deu em um cenário marcado pela judicialização da saúde no Brasil e deve possuir um papel importante em proporcionar maior transparência às análises de demandas de incorporação de tecnologias no SUS e em ampliar a participação da sociedade por meio das consultas públicas (12).

Com o objetivo de analisar as demandas por incorporações de medicamentos, esse trabalho se propõe a responder três argumentações:

a) Quais são os principais segmentos sociais que demandam à CONITEC análises por incorporação de medicamentos no SUS? 
b) Quais são os principais segmentos que participam com contribuições para as consultas públicas realizadas para a incorporação de medicamentos no SUS?

c) É possível, a partir dos dados obtidos, inferir possíveis influências dos diversos segmentos envolvidos no processo de incorporação?

\section{Metodologia}

Foi realizado um estudo por meio de pesquisa descritiva quantitativa, a respeito da participação de segmentos sociais nas demandas e consultas públicas realizadas no período de 2012 a 2014, sobre incorporação de medicamentos no SUS.

Os dados se encontraram livres e desembaraçados, com acesso público no sítio: (http://conitec.gov.br/).

A coleta de dados foi realizada utilizando os relatórios das demandas de análises de incorporação de medicamentos que estão disponíveis no sítio: http://conitec.gov.br/index.php/tecnologias-em-avaliacao. Foram excluídas todas as tecnologias em saúde que não são classificadas como medicamentos (procedimentos e produtos para saúde). Os dados foram compilados em uma planilha em Excel (versão 2013) e foram contabilizadas quantas demandas foram encaminhadas a CONITEC em todo o período estudado (2012 a 2014), totalizando 214 demandas de medicamentos do total de 342 demandas por tecnologias em saúde.

Filtrando apenas o quantitativo de demandas por medicamentos, foram identificados quais foram os demandantes por análises de incorporação sendo encontrados tais segmentos: empresas/laboratórios farmacêuticos (nomes das empresas demandantes), gestores estaduais, gestor federal (áreas técnicas do Ministério da Saúde), associação de pacientes, profissional de saúde, órgão do Poder Judiciário e, comitês e sociedades médicas.

Para análise das consultas públicas, foi realizado um levantamento por meio dos relatórios das consultas públicas para identificar a participação dos segmentos sociais, que estão disponíveis no sítio: http://conitec.gov.br/index.php/consultas-publicas. Foram encontrados tais segmentos identificados nas consultas públicas: empresa, empresa fabricante da tecnologia avaliada, Secretaria Municipal de Saúde, Secretaria Estadual de 
Saúde, Ministério da Saúde, associação de pacientes, profissional de saúde, instituição de ensino, instituição de saúde, sociedade médica, paciente/usuário, familiar ou amigo de usuário e outro.

Os dados foram compilados em planilha em Excel (versão 2013) e foram contabilizadas as consultas públicas realizadas de 2012 a 2014, bem como a participação dos segmentos em cada consulta realizada. Foram encontradas 65 consultas públicas sobre incorporações de medicamentos, sendo dessas 14 realizadas em 2012, 12 consultas em 2013 e 29 realizadas em 2014.

Os dados foram plotados em gráficos e tabelas para que fossem comparados e assim se pudessem fazer uma análise quantitativa dos dados observados.

\section{Resultados e Discussões}

\section{Análise das demandas por incorporações de medicamentos no SUS}

Desde a criação da CONITEC foram realizadas 342 demandas para análise de incorporações de tecnologias no SUS, sendo dessas 214 demandas por medicamentos. É importante destacar que as análises solicitadas pelos demandantes podem ser para incorporação, exclusão e/ou ampliação de uso de determinada tecnologia.

Ao analisar o número de demandas para análise de incorporação de medicamentos, constatou-se que os demandantes foram empresas/laboratórios farmacêuticos que detém o total de 94 demandas; associação de pacientes que apresentaram um total de 8 demandas; órgãos do Poder Judiciário (Advocacia Geral da União, Defensoria Pública, Justiça Federal e Ministério Público) totalizando 8 demandas; Comitês e Sociedades Médicas com 3 demandas; Gestores de saúde dos estados e Ministério da Saúde responsáveis por 100 demandas; e profissionais de saúde com 1 demanda.

Os dados representados no gráfico 1 demonstram que os principais demandantes por incorporações são o Ministério da Saúde e as empresas/laboratórios farmacêuticos. 
Gráfico 1 - Número de demandas totais de análises de incorporação de medicamentos por segmentos social no período de 2012 a 2014.

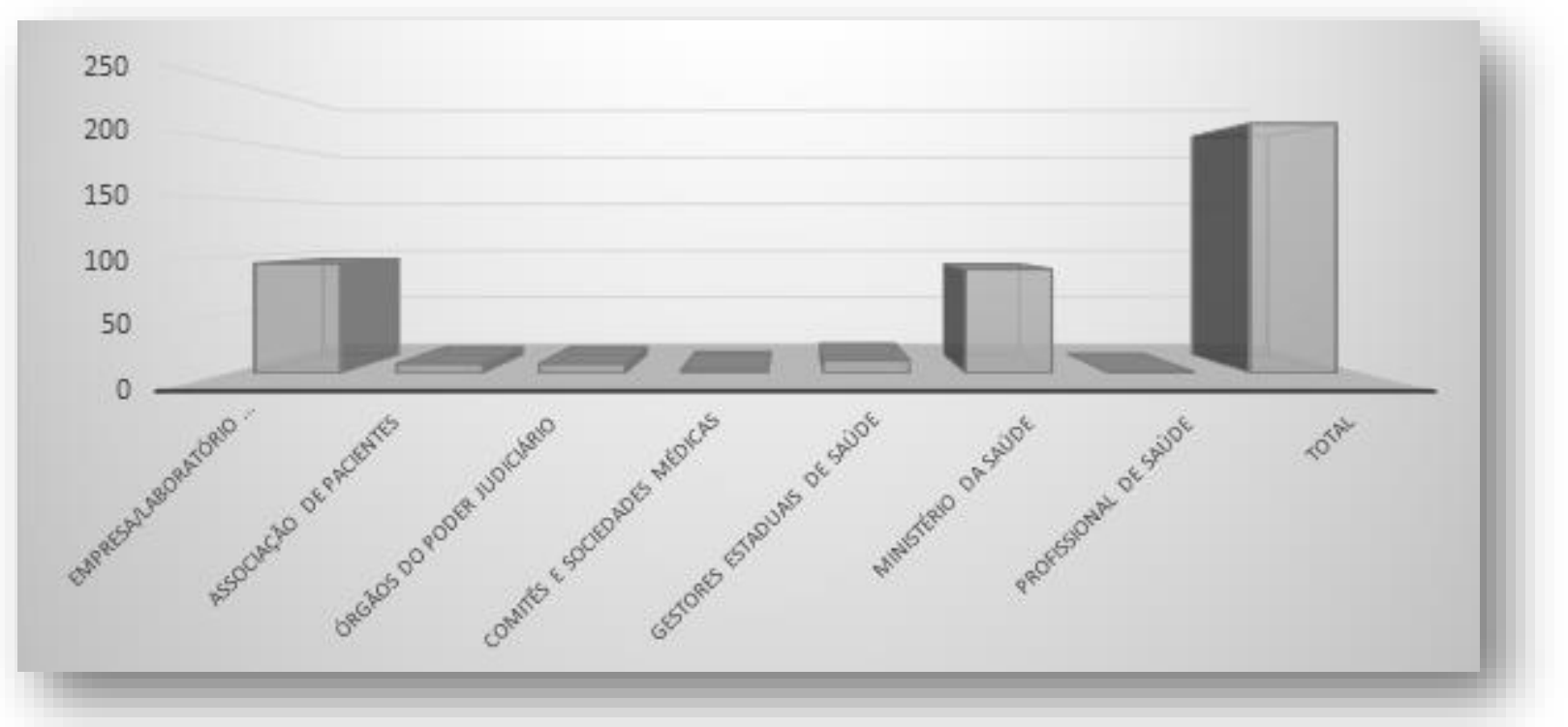

Fonte: CONITEC, 2014.

O único demandante que apresentou solicitação de análise de exclusão foi o Ministério da Saúde, que dentre as 89 demandas de análises, 16 solicitações de estudos foram de exclusão de medicamentos já incorporados no SUS. É possível observar nos dados apresentados, que não há a participação de usuários do SUS (isoladamente) na apresentação de demandas à CONITEC. Apenas o segmento de associação de pacientes, por se constituir em um movimento social organizado e com conhecimento específico por uma determinada doença ou agravo à saúde, possui alguma participação nesse processo.

\section{Análise da situação das demandas por incorporação de Medicamentos no SUS}

Após o recebimento da demanda, a CONITEC atualiza a situação de análise para cada uma das demandas encaminhadas. Essa etapa é bastante importante para se compreender, quais demandas de fato resultaram em incorporações, conforme apresentado na tabela 1.

Os dados apresentados demonstram que aproximadamente $18 \%$ das demandas apresentadas foram rejeitadas por não apresentarem conformidades com a documentação que é exigida no processo de apresentação de proposta para incorporação. Isso pode refletir 
uma dificuldade na obtenção de informações sobre o processo durante a etapa de organização da documentação e/ou despreparo técnico por parte dos proponentes.

Tabela 1 - Situação das demandas por incorporação de tecnologias na CONITEC/MS realizadas até outubro/2014.

\begin{tabular}{|c|c|c|c|c|c|c|c|c|}
\hline Demandante & $\begin{array}{l}\text { Total de } \\
\text { Demanda } \\
\text { s }\end{array}$ & $\begin{array}{c}\text { Análise/aguardand } \\
\text { o apreciação final } \\
\text { para publicação }\end{array}$ & $\begin{array}{l}\text { Encerradas a } \\
\text { pedido do } \\
\text { demandante }\end{array}$ & $\begin{array}{l}\text { Tecnologias } \\
\text { Incorporada } \\
\text { s no SUS }\end{array}$ & $\begin{array}{c}\text { Tecnologias } \\
\text { não } \\
\text { Incorporadas } \\
\text { no SUS }\end{array}$ & $\begin{array}{l}\text { Tecnologia } \\
\text { fora do } \\
\text { Escopo da } \\
\text { CONITEC }\end{array}$ & $\begin{array}{c}\text { Recusadas } \\
\text { por não } \\
\text { conformidade } \\
\text { formal de } \\
\text { documentação }\end{array}$ & $\begin{array}{c}\text { Exclusões } \\
\text { de } \\
\text { Tecnologia }\end{array}$ \\
\hline $\begin{array}{l}\text { Empresa/Lab. } \\
\text { Farmacêutico }\end{array}$ & 94 & 10 & 5 & 12 & 36 & 1 & 30 & - \\
\hline $\begin{array}{l}\text { Associação de } \\
\text { Pacientes }\end{array}$ & 8 & 8 & - & 3 & - & - & 5 & - \\
\hline $\begin{array}{l}\text { Órgãos do } \\
\text { Poder } \\
\text { Judiciário }\end{array}$ & 8 & 3 & - & 2 & 3 & - & - & - \\
\hline $\begin{array}{l}\text { Comitês e } \\
\text { Sociedades } \\
\text { médicas }\end{array}$ & 3 & - & - & - & - & - & 3 & - \\
\hline $\begin{array}{l}\text { Gestores } \\
\text { Estaduais de } \\
\text { Saúde }\end{array}$ & 11 & 6 & - & - & 5 & - & - & - \\
\hline $\begin{array}{l}\text { Ministério da } \\
\text { Saúde }\end{array}$ & 89 & 27 & 9 & 33 & 4 & - & - & 16 \\
\hline $\begin{array}{l}\text { Profissional de } \\
\text { Saúde }\end{array}$ & 1 & - & - & - & 1 & - & - & - \\
\hline Total & 214 & 54 & 14 & 50 & 49 & 1 & 38 & 16 \\
\hline
\end{tabular}

Fonte: CONITEC, 2014.

Os resultados encontrados também revelam que o segmento que possui a maior parte de suas demandas resultante em pareceres favoráveis à incorporação é o Ministério da Saúde. Apesar do expressivo número de propostas realizadas pelo segmento representado pelas empresas e laboratórios farmacêuticos refletido no número total de demandas interpostas no período, esse segmento possuiu 38\% das suas propostas com pareceres desfavoráveis à incorporação e $32 \%$ rejeitadas por não conformidade formal à documentação.

Mas, ainda que esses números permitam uma possível inferência de que as exigências da CONITEC no processo de avaliação para incorporação estejam mais rígidas, é válido ressaltar que a representatividade no número de demandas consideradas favoráveis pela CONITEC é majoritariamente desses dois segmentos (Ministério da Saúde e Laboratórios Farmacêuticos). 


\section{Análise das Consultas Públicas realizadas pela CONITEC entre 2012 a 2014}

A etapa de consulta pública para avaliação das tecnologias a serem incorporadas no SUS é necessária em respeito à participação social e à transparência nesse processo. Ao analisar as consultas públicas realizadas pela CONITEC nos anos de 2012, 2013 e 2014, percebeu-se que as contribuições expressamente identificadas como usuários/pacientes são bastante incipientes, pois representam ao longo dos três anos uma participação em apenas 04 das 50 consultas públicas que tiveram seus participantes devidamente identificados.

Um dado importante avaliado é que durante o ano de 2012, 14 consultas públicas não apresentaram, em seus relatórios, a identificação de seus contribuintes, ou seja, as contribuições aparecem sem a descrição de quem as realizou. Essa falha na identificação dos segmentos prejudica a transparência das consultas públicas, bem como a contabilização dos dados para o estudo.

Outro dado relevante é que durante o período dos anos estudados, 09 consultas públicas não tiveram nenhum tipo de contribuição dos segmentos sociais, conforme apresenta o gráfico 2 .

Gráfico 2 - Número de consultas públicas por ano realizadas sem identificação dos contribuintes ou sem contribuição dos segmentos sociais no período de 2012 a 2014.

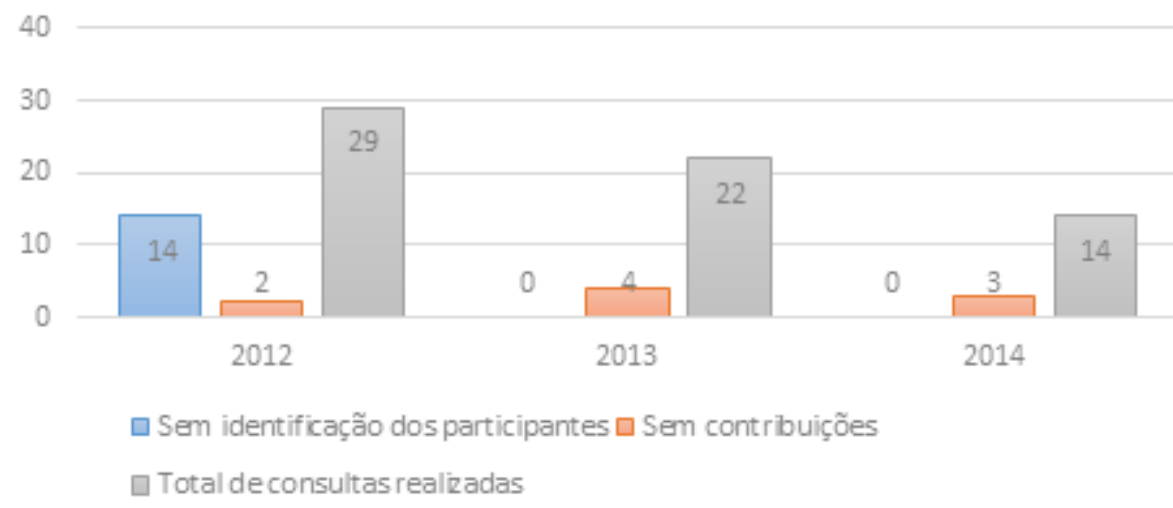

Fonte: CONITEC, 2014.

Considerando o número de consultas com as contribuições devidamente identificadas, os resultados demonstraram que o segmento empresa ou empresa fabricante da tecnologia avaliada são os que mais participam das consultas públicas. Das 50 consultas, 
as empresas estão presentes em 37 delas. A maioria das vezes em que não há sua participação, se deu em consultas em que não houve nenhuma contribuição.

Gráfico 3 - Presença dos segmentos sociais nas consultas públicas por identificação do contribuinte em cada ano no período de 2012, 2013 e 2014.

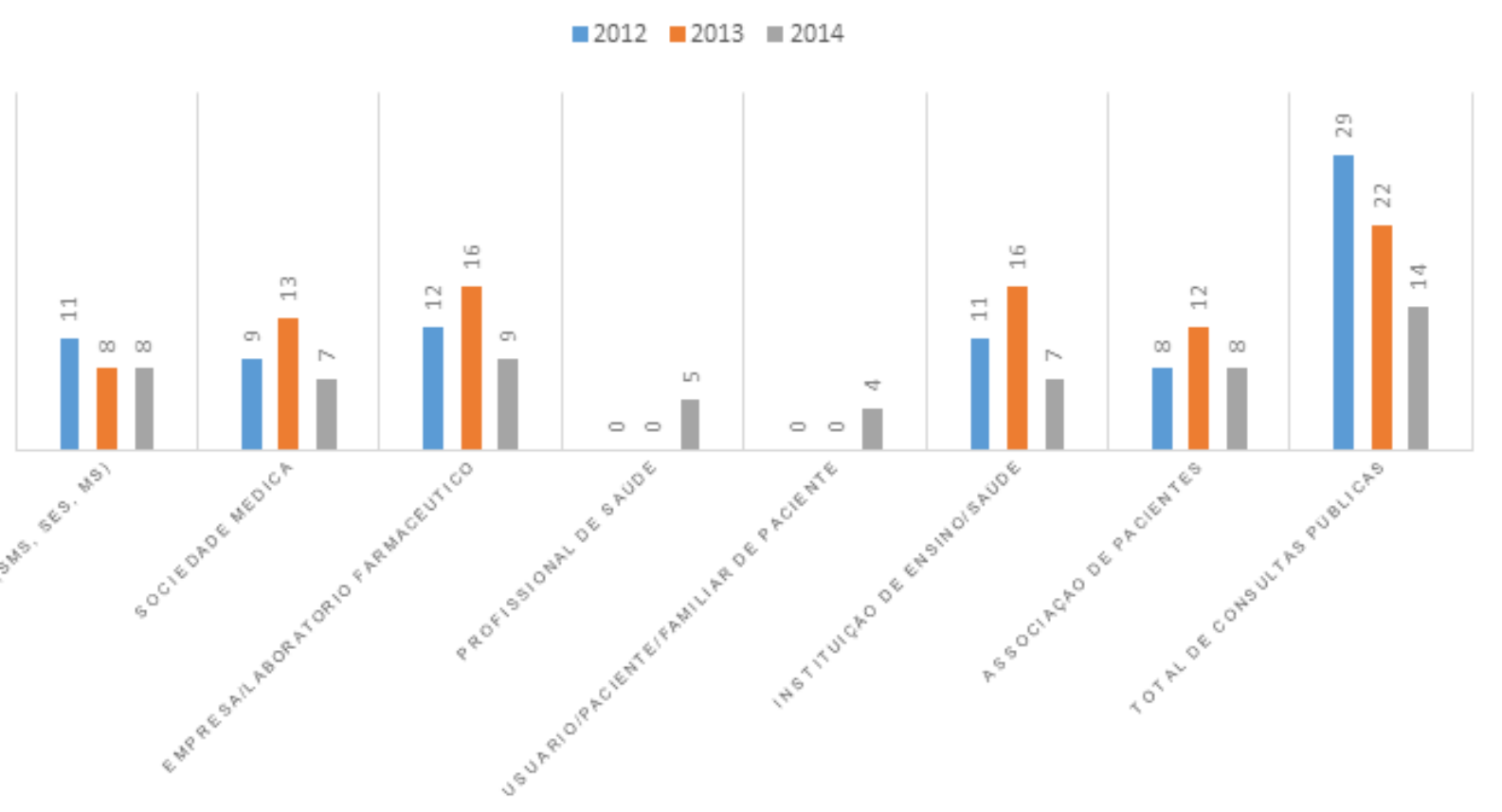

Fonte: CONITEC, 2014.

Considerando todos os dados coletados, percebeu-se que algumas informações apresentam alguns vícios que precisam ser destacados. Os relatórios das consultas apresentam falhas na identificação dos seus participantes, o que prejudica a garantia da transparência do processo. A possibilidade de identificação do segmento como 'outro' para realizar as contribuições nas consultas não permite analisar quem de fato contribuiu para a avaliação de incorporação.

Além disso, algumas identificações foram feitas de maneira inadequada, a exemplo da figura 2, na qual o segmento se identifica como empresa, no entanto realiza uma contribuição que deveria ser de um suposto usuário/paciente do SUS ou familiar/amigo de paciente. 
Figura 2 - Incoerências nas identificações dos segmentos sociais das consultas públicas realizadas pela CONITEC.

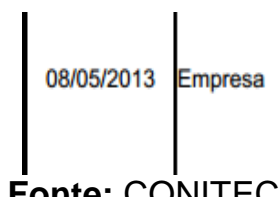

Estou com um parente próximo com esclerose múltipla, que falhou o tratamento com interferona. O mesmo teve que entrar na ustiça para conseguir natalizumabe, já que se nao utilizasse o mesmo poderia ter surto incapacitaste devido a gravidade da sua doença. A indicaçăo em bula do natalizumabe e na falha de um tratamento e nao entendo o motivo do ministério exigir 2.

Fonte: CONITEC, 2014.

A CONITEC, desde sua criação, tomou 115 decisões a respeito de análises de incorporação de medicamentos, conforme os resultados encontrados. Os dados demonstram uma prevalência dos segmentos correspondentes às empresas e laboratórios farmacêuticos e das áreas técnicas do Ministério da Saúde como principais demandantes de avaliações.

Algumas questões podem ser atribuídas a maior participação desses segmentos nesse processo, tais como: assimetria de informação, pois quando comparados aos demais segmentos, o laboratório farmacêutico fabricante detém maior conhecimento técnico específico sobre a tecnologia a ser avaliada e os interesses econômicos provocam uma contínua busca das empresas por informação a respeito das incorporações; as áreas técnicas demandantes do Ministério da Saúde possuem maior domínio sobre as etapas do processo de avaliação de incorporação, reduzindo as dificuldades para encaminhar as demandas a CONITEC, principalmente no tocante às possíveis recusas por não conformidade formal de documentação; a incipiente difusão da informação de maneira mais ampla para que segmentos sociais como usuário/paciente possam participar mais efetivamente das avaliações de incorporações.

Das 94 demandas apresentadas pelas indústrias farmacêuticas, 12 foram incorporadas ao SUS e 30 dessas foram recusadas por não conformidade documental. Essas 12 incorporações correspondem a 24\% das decisões da CONITEC a favor da incorporação de medicamentos, ficando atrás apenas das demandas aceitas das próprias áreas técnicas do Ministério da Saúde, que representam $66 \%$ das incorporações realizadas nos últimos três anos.

Outro dado que evidencia a maior probabilidade de uma proposta de incorporação, realizada por uma determinada área do Ministério da Saúde à CONITEC, ser aprovada, é que durante todo o período avaliado não houve nenhuma recusa por não conformidade 
documental, ao contrário de segmentos como associação de pacientes, sociedades médicas e das demandas dos laboratórios farmacêuticos.

Esse estudo também permitiu a constatação da baixa participação de profissionais de saúde e usuários tanto como demandantes quanto contribuintes nas consultas públicas. As associações de pacientes por se tratarem de um movimento socialmente organizado conseguem maior envolvimento e oportunidade na participação, mas isoladamente, 0 indivíduo enquanto usuário, paciente ou familiar de algum paciente acometido por determinada doença ou agravo ainda não conseguiu se inserir de maneira perceptível no processo.

Apesar das avaliações de tecnologias em saúde necessitarem de conhecimento técnico específico, isso não significa que as propostas não possam ser realizadas por segmentos sociais que não detenham a total dimensão de conhecimento necessário a respeito da tecnologia pleiteada, bastando a mera expectativa de necessidade que determinada tecnologia represente para o usuário para que ele se sinta parte do processo propositivo.

Apesar da previsão legal da participação social no processo administrativo de incorporações de tecnologias no SUS, observa-se nesse cenário, a relevância de execução de uma melhor gestão e disseminação do conhecimento para que haja o efetivo exercício da participação social na obtenção do direito integral à saúde.

As instituições de ensino e pesquisa e as instituições de saúde aparecem de maneira significativa nas consultas públicas, porém as últimas quando representadas pelos seus gestores municipais e estaduais para realizar as demandas para avaliação de determinado medicamento, seja para sua exclusão, ampliação de uso ou incorporação não se mostram representativas.

E aqui nesse ponto, é válido ressaltar a importância das contribuições dos segmentos para a revisão da Relação Nacional de Medicamentos (RENAME), considerando a necessidade de demandas por estudos de exclusão de medicamentos que estão obsoletos por inadequação de uso, por substituição por um novo medicamento, por notificações de eventos adversos, dentre outros fatores. Os gestores estaduais e municipais possuem papel importante na identificação desses itens que muitas vezes apenas oneram a lista de investimentos em tecnologias com recursos federais para a saúde, mas que já não atendem às necessidades da população. 
Novaes et al (7), em seu estudo sobre o uso de avaliação de tecnologias em saúde nos processos de incorporações de tecnologias no SUS, identificaram desafios para que o campo de avaliação de tecnologias tenha impactos no SUS, tais como a necessidade de ampliação de foco para as ações de exclusão de medicamentos, uma maior intersecção com a gestão estadual, municipal e a saúde suplementar, bem como a autonomia e a transparência nos processos institucionais.

Outro desafio colocado no mesmo estudo é a necessidade de imprimir uma ação ativa na definição de prioridades e na influência exercida pelo mercado privado. Os dados avaliados neste trabalho corroboram para mais uma vez ratificar os desafios levantados pelo estudo de Novaes et al (7).

Ainda é válido reafirmar que apesar da regulação da incorporação de tecnologias do SUS tenha apresentado avanços principalmente quanto à edição de atos normativos que regulamentam a atuação da CONITEC e da participação social, permanece o constante desafio da efetivação da participação e do controle social no âmbito da Política Nacional de Incorporação de Medicamentos no Brasil.

\section{Considerações finais}

Constatou-se, portanto, que as empresas e laboratórios farmacêuticos são os que possuem maior participação tanto nas demandas por incorporações de medicamentos quanto como contribuintes nas consultas públicas. Porém, os demandantes que obtiveram maior número de análises que resultaram em incorporações efetivas foram as áreas técnicas do próprio Ministério da Saúde, o que reflete uma necessidade de maior disseminação do conhecimento e do fortalecimento da participação social no SUS, pois os demais segmentos como usuários e profissionais de saúde ainda demonstram uma participação incipiente no processo.

Apesar da normatização da CONITEC como um espaço colegiado que conta com representantes de vários segmentos, ainda pode-se questionar sua efetividade quanto instância deliberativa que assegure de fato a participação social, já que gestores federais e representantes dos interesses de mercado são os que possuem mais conhecimento técnico para que suas demandas sejam incorporadas no SUS. 
Além disso, mesmo com a garantia da realização prévia de consulta pública, que é uma ferramenta imprescindível para a transparência do processo, esta apresenta falhas importantes, como por exemplo na identificação dos contribuintes, que comprometem seu principal objetivo que é garantir a participação social e a divulgação das informações.

Outro aspecto relevante, é que a grande participação do segmento da indústria farmacêutica nos permite refletir sobre o grande interesse desse segmento nas incorporações de medicamentos no sistema de saúde no Brasil. A incorporação de medicamentos está intrinsecamente relacionada à inserção de tecnologias de ponta no sistema de saúde, que trazem inúmeros benefícios para a sociedade, mas estão imbuídos de riscos que precisam ser avaliados e também resultam em alto impacto financeiro para o sistema de saúde. E um questionamento importante é que será que esses produtos sempre representam inovação tecnológica? Sendo assim, mais estudos são necessários utilizando os critérios do método de avaliação de tecnologia em saúde nas análises da CONITEC para que se possa avaliar uma possível influência das relações de mercado nas incorporações de medicamentos no Brasil.

Pode-se verificar ainda que, apesar da percepção social sobre a saúde como um objeto de consumo refletir na judicialização do direito ao acesso de medicamentos, não se consegue enxergar, de forma suficiente, a reivindicação do usuário por melhores condições de acesso e por mais inovações tecnológicas que visem garantir melhores tratamentos e condições de saúde, em sua forma de participação social organizada e institucionalizada com a utilização dos devidos instrumentos legais que devem ser garantidos pelas políticas de saúde.

Por fim, apesar das avaliações de tecnologias exigirem um conhecimento técnico específico, elas não podem representar barreiras para a inserção dos diversos segmentos sociais - principalmente os profissionais de saúde e usuários do SUS -, para que a judicialização da saúde não seja a única alternativa viável de usuários reivindicarem do SUS a efetivação do direito de acesso aos medicamentos.

Faz-se necessário divulgar e discutir amplamente a Política Nacional de Incorporação de Medicamentos no Brasil para garantir a participação e o controle social para que se proteja o direito à saúde. 


\section{Referências}

1 - Brasil. Constituição (1988). Constituição da República Federativa do Brasil. Brasília, DF: Senado Federal; 2001.

2 - Capucho HC et al. Incorporação de tecnologias em saúde no Brasil: novo modelo para o Sistema Único de Saúde. Boletim do Instituto de Saúde. 2012 [Acesso em 30 out 2014]. Disponível em:

http://conitec.gov.br/images/Artigos Publicacoes/IncorporacaodeTecnologia emSaude Br asil.pdf

3 - Ministério da Saúde. Nova Comissão Nacional de Incorporação de Tecnologias de Saúde e impacto ao Sistema Único de Saúde. Rev. Saúde Pública. 2011 [Acesso em 30 out 2014], 45(5): 993-996. Disponível em:

http://www.scielo.br/scielo.php?script=sci arttext\&pid=S0034-89102011000500024

4 - Gadelha CAG. Desenvolvimento de Saúde. 2012 [Acesso em 01 nov 2014]. Disponível em: http://conitec.gov.br/images/Artigos Publicacoes/Desenvolvimento-

Saude CarlosGadelha.pdf

5 - Santos VCC. As análises econômicas na incorporação de tecnologias em saúde: reflexões sobre a experiência brasileira [Dissertação]. Rio de Janeiro: Escola Nacional de Saúde Pública, Fundação Oswaldo Cruz; 2010.

6 - Brasil. Ministério da Saúde. Política Nacional de Gestão de Tecnologias em Saúde. 2010. [Acesso em 29 out 2014]. Disponível em:

http://bvsms.saude.gov.br/bvs/publicacoes/politicanacional gestao tecnologias saude.pdf

7 - Novaes HMD, Elias FTS. Uso da avaliação de tecnologias em saúde em processos de análise para incorporação de tecnologias no Sistema Único de Saúde no Ministério da Saúde. 2013 [Acesso em 1ํㅡov. 2014], 29(1): s7-s16. Disponível

em: http://www.scielo.br/scielo.php?pid=S0102-311X2013001300002\&script=sci arttext

8 - Oliveira NA, SANTOS, Lenir. Gestão do conhecimento na saúde. Direito sanitário: Oportuna discussão via coletânea de textos do 'blog Direito Sanitário: Saúde e Cidadania'. Brasília: ANVISA, CONASEMS, CONASS; 2012. [Acesso em 29 out. 2014]. Disponível em: http://www.anvisa.gov.br/hotsite/anvisa/publicacoes/livrodireitosanitario.pdf

9 - Chomsky N. Segredos, Mentiras e Democracia. Brasília: Editora Universidade de Brasília; 1997.

10 - Brasil. Lei 12.401, de 28 de abril de 2011. Altera a Lei no 8.080, de 19 de setembro de 1990, para dispor sobre a assistência terapêutica e a incorporação de tecnologia em saúde no âmbito do Sistema Único de Saúde - SUS. Diário Oficial [da] República Federativa do Brasil. Brasília, DF, 29 de abril de 2011. [Acesso em 1ํㅡ nov 2014]. Disponível em: http://www.planalto.gov.br/ccivil 03/ Ato2011-2014/2011/Lei/L12401.htm

11 - Brasil. Decreto 7.646, de 21 de dezembro de 2011. Dispõe sobre a Comissão Nacional de Incorporação de Tecnologias no Sistema Único de Saúde e sobre o processo 
administrativo para incorporação, exclusão e alteração de tecnologias em saúde pelo Sistema Único de Saúde - SUS, e dá outras providências. Diário Oficial [da] República Federativa do Brasil. Brasília, DF, 22 de dezembro de 2011. [Acesso em 1 nov 2014]. Disponível em: http://www.planalto.gov.br/ccivil 03/ Ato20112014/2011/Decreto/D7646.htm

12 - Comissão Nacional de Incorporação de Tecnologias no SUS. Brasília: 2014. [Acesso em 1 - nov 2014]. Disponível em: http://conitec.gov.br/index.php/fluxo-de-incorporacao-detecnologias-no-sus 